\title{
4 Bewusste und leidenschaftliche Führungskultur
}

\author{
Dorothea Galuska
}

\section{Die Anforderungen an eine moderne Führungskraft}

Wie schwierig und teilweise überfordernd unsere heutigen Führungsaufgaben sind, zeigt uns die humorvolle Beschreibung für die Idealbesetzung eines neuen Polizeipräsidenten:

„Die ideale Führungskraft braucht: die Würde eines Erzbischofs, die Selbstlosigkeit eines Missionars, die Beharrlichkeit eines Steuerbeamten, die Erfahrung eines Wirtschaftsprüfers, die Arbeitskraft eines Kulis, den Takt eines Botschafters, die Genialität eines Nobelpreisträgers, den Optimismus eines Schiffbrüchigen, die Findigkeit eines Rechtsanwalts, die Gesundheit eines Olympiakämpfers, die Geduld eines Kindermädchens, das Lächeln eines Filmstars und das dicke Fell eines Nilpferds".

... - und dann auch noch Leidenschaft, Begeisterung, Inspiration, Balancierung, Blick für die Realität, Improvisationstalent ...? Sind wir Tänzer mit tausend Köpfen, tausend Armen und Beinen? Manchmal fühlen wir uns vielleicht so und manchmal fühlen wir uns richtig gut so, kompetent und kraftvoll. Und an anderen Tagen fehlen uns mindestens 1o bis 1oo Arme und sogar unser einer Kopf ist benebelt, das Gleichgewicht stimmt nicht und tanzen mögen wir schon gar nicht. Und dann?

„Auch das geht vorüber“ - sagen spirituelle Menschen, „Vielleicht hilft ein Freund?“sagen soziale Menschen oder „Versuch es doch mit Selbstmanagement“ - könnte der Tipp von Beratern und Coaches sein.

Wie können wir in unserer Führung offen bleiben - auch in diesen unbequemen Zuständen - sie vielleicht sogar wachstumsfördernd nutzen - denn sie spiegeln eine 
innere Grenze, ein inneres Thema. Nicht der uns so vertraute und aktive Macher führt hier unser Erleben, sondern die Empfindsamkeit der eigenen Seele.

Wenn wir akzeptieren, dass Führung neben der Berufung auch ein eigener Beruf ist, für den wir zum Erlernen mindestens so viel Zeit investieren müssen wie in den fachlichen Grundberuf, dann werden wir uns Führungshandwerkszeug, ManagementKnow-how und komplexe Organisationsweisen aneignen. Schwerer tun wir uns meist mit dem Gebiet der Mitarbeiterführung v.a. im Erlernen der sogenannten „Softskills“ und die größte, herausforderndste und am häufigsten übersehene Baustelle ist und bleibt unsere Selbstführung.

\section{Selbstführung: Ein Mangel an eigener Bewusstheit}

Oft widmen wir uns der Selbstführung erst nach erheblichen Überforderungs- und Erschöpfungserlebnissen, manchmal erst bei deutlicher körperlicher und seelischer Erkrankung. Wie schade! Denn es gibt in uns Menschen eine nicht immer laute und fordernde, aber meist klare Stimme - ich würde sagen: die Stimme unserer Seele die uns auf wunderbare Weise inneres Geleit anbietet und uns innere Navigation und Führung anträgt.

Warum hören wir sie nicht? Yehudi Menuhin (1987) merkt dazu an:

Warum eigentlich fürchten wir die Stille?

Weil wir den Spiegel, den sie uns vorhält, nicht ertragen können, weil wir verlernt haben, wirklich tief ehrlich zu sein, mit anderen und auch mit uns selbst.

Die innere Stimme hat uns auch heute, in diesem tönenden, lauten, ja ich möchte sagen, gewalttätigen Leben nicht verlassen.

Sie ist auch nicht leiser geworden, nur wir sind lauter geworden und stellen uns taub aus Angst vor der Konsequenz, aus Angst, einfach zu leben und zu denken.

Wir müssen erneut Mut und Vertrauen erlernen, den Mut auf unsere eigene innere Stimme zu hören, und das Vertrauen, ihr zu folgen, denn alles laute Betäuben oder gar Abtöten ist nur ein zielloser Ausweg, der in einer Sackgasse anstatt in der Weite der echten Freiheit enden muss.

In uns Führungskräften sind oft Machtwünsche, Ehrgeiz oder, „sich selbst etwas beweisen wollen" die treibenden Kräfte, die uns aus der Verankerung unserer eigenen Tiefe an die Oberfläche spülen. Manchmal sind es auch soziale Wünsche von Ausgleich nach Gerechtigkeit, Heilung oder Vergebung, die eigentlich tief in uns verankert sind, uns aber dennoch im Handeln von uns weg treiben. Im Strudel der „To-doListen“, im Korridor unserer Zielvereinbarungen und unter dem Ergebnisdruck der Jahresbilanzen zieht sich die Seele nach Innen zurück und wartet in ihrer Dialogbereitschaft auf die nächste entspanntere Begegnung. Und doch ist sie wach, begleitet unseren Management-Tanz, registriert unsere An- und Verspannungen, ist sich unserer Verbiegungen, Versuchungen und Verrutschungen aus der Wahrhaftigkeit sehr bewusst. Sie ist liebevoll, geduldig, verzeihend - aber nicht unbegrenzt. In ihrer unserer Persönlichkeit zugewandten Seite versucht sie zunächst behutsame, später deutlichere Signale zur Umkehr und Innenwendung zu senden: Sie benutzt z.B. leich- 
te Unruhe, Spannung im Herzen oder Brustbein, Kopfschmerzen oder sich nicht mehr konzentrieren können als ihre Sprache. Wir könnten sie als Botschaften zum Innehalten in unser Bewusstsein übersetzen.

\section{Das Potenzial bewusster Führung}

Ich lese sie gern als Rückruf und als Einladung aus dem nach außen gerichteten Schaffenszyklus in den rezeptiven nach innen gerichteten Zyklus einzukehren. Daher stammen sicher auch die vielen populären auf Balancierung ausgerichteten Seminare mit Entspannungstechniken, Selbstmanagement, Meditation, Fasten, Wandern, Schweigen - oder der immer wiederkehrende Wunsch nach Auszeit und Urlaub. Das ist weise und oft sehr sinnvoll.

Und doch erlaube ich mir die Frage, ob es neben diesen längerfristigen Zyklen, die der Gesunderhaltung dienen, nicht auch alltägliche Durchdringungen von Seele und Management, von Seele und Führung gibt? Müssen wir mit dem Seelen-Dialog wirklich warten, bis es Zeit ist für die nächste Meditation, den nächsten Urlaub oder wenigstens das nächste Wochenende? Ist es wirklich gesetzt, dass wir zwischen „work“ und „life“, unserer Arbeit und dem Rest unseres Lebens balancieren, zwischen bezahltem Schmerzensgeld für unsere Arbeit und der sogenannten fast scherzhaften „Quality-time“, unserer Freizeit?

Manchmal, nicht oft, aber durchaus erlebt, verkehrt sich diese Priorisierung auch angesichts einigermaßen erfüllender Arbeitsbedingungen und eher unglücklicher privater Entwicklungen. Dann wird die Führungsaufgabe schon mal zur Flucht vor dem anstehenden Beziehungsgespräch oder dem dauerhaften Konflikt zu Hause!

Ich fürchte aber, dass diese Betrachtung die Schere und Spannung in unserer Innenwelt eher vertieft oder zumindest zementiert. Unsere polarisierende „Entweder/oder“Sprache entlarvt uns hier.

- Wie wäre es, mitten in unserer Führungsverantwortung, die Seele offen zu halten?

- Uns die Zeit des Innehaltens, der Vergegenwärtigung des Lebens inmitten unseres Tuns und Gestaltens zu gönnen?

Es gibt hierzu Ideen und Ansätze im Sinne einer „achtsamen Führung“. Einige von uns experimentieren bereits mit Phasen von Stille, dem Vorlesen philosophischer oder spiritueller Weisheitstexte, der Integration der inneren Haltung hinein in die Momente des Arbeitens. Aber wir sind noch sehr zaghaft in unseren Unternehmen. Wer von uns darf dort seine zarten, verletzlichen, ringenden Seiten zeigen oder sie wenigstens erwähnen? Wer fürchtet nicht, dass ihm diese Offenbarung als Autoritätsverlust oder als Schwäche gedeutet wird? In welchen Unternehmen werden hitzige, sich im Kreis drehende Debatten von einer „Glocke der Achtsamkeit“ unterbrochen und die Teilnehmer zum Innehalten und Desidentifizieren von Positionen und Meinungen eingeladen?

Welche Führungskraft stellt im Mitarbeitergespräch die auf der Checkliste stehende Frage: Sind Sie mit Ihrem Herzen und Ihrer Leidenschaft bei Ihrer Arbeit? Und wenn nicht, was können wir ändern, damit dies möglich wird? Und ganz heikel: kann ich selbst in Situationen eigener Ambivalenz, eigener Verletzlichkeit oder aktuellen Leidens auf das Mitgefühl meiner Kollegen und besonders das von mir selbst vertrauen? 
In vielen Fällen sind wir selbst unsere schärfsten Kritiker und Richter. Und schlimmer noch: Wir kritisieren nicht unsere Handlungen, sondern ganz gezielt unseren Wert, unseren Selbstwert. Wir kränken und schwächen uns selbst. Allein wenn wir lernen, diese Angriffsrichtungen zu unterscheiden, d.h. den tiefen Wert in uns selbst und anderen wirklich unangetastet zu lassen und dabei gleichzeitig kritisch in der Sache zu bleiben, ist ein Meilenstein in der Führungs- und Selbstführungskompetenz geschehen.

Weder werden wir uns unnötigerweise mit Selbstvorwürfen quälen, noch werden wir unseren Mitarbeitern gegenüber übermäßig hart oder vorwerfend auftreten. Ein Buch-Tipp für dieses Thema trägt den schönen Titel: „Befreiung vom inneren Richter-die Intelligenz der Seele erkennen“ von Byron Brown (2004).

\section{Der Wert bewusster Mitarbeiterführung}

Führungskräfte sollten sich grundsätzlich vergegenwärtigen, dass ihre Hauptzielgruppe ihre eigenen Mitarbeiter sind. Diese leiden oft unter ähnlichen Belastungssituationen und sehr oft auch unter den nicht bewussten Themen und Beziehunskonflikten ihrer Vorgesetzten. Wenn die Führungskraft ihre „Hausaufgaben“ an Bewusstwerdung gemacht hat, entspannen sich häufig nicht nur einzelne Mitarbeiter, sondern auch ganze Teamdynamiken. Daher ist es erforderlich, Führungskräfte für das Thema der psychosozialen Belastungen zuerst einmal zu sensibilisieren und ihnen ein Basiswissen darüber zu vermitteln, welche Bedeutung ihr Führungsverhalten, die Arbeitsbedingungen und die Unternehmenskultur für die psychosoziale Gesundheit ihrer Mitarbeiter besitzen.

Die Führungskraft kann zum authentischen Vorbild werden und Selbstführung vorleben und anleiten. In dieser Funktion übt sie eine pädagogische Rolle für das Thema „Resilienz“ aus. Neben den fachlichen Kompetenzen können Führungskrafte auch auf die Kommunikations- und Kooperationsfähigkeiten ihrer Mitarbeiter achten, sowie ein proaktives Gesundheitsverhalten fördern. Wenn wir in einer Führungsverantwortung auch unsere grundlegenden Werte und die unserer Mitarbeiter reflektieren und überprüfen, und diese auf eine gemeinsame Passung hin untersuchen, können wir sowohl cokreatives Potenzial als auch mögliche Konfliktpunkte erkennen. Eine entscheidende Bedeutung dabei besitzt die Fähigkeit zu Empathie. Empathie ist Einfühlung in die Welt eines Anderen und basiert auf der Fähigkeit zum Perspektivwechsel und zum Mitgefühl. Die innere Situation eines anderen Menschen zu spüren und darauf verständnisvoll und sachgerecht reagieren zu können, macht die eigentliche Dialogfähigkeit aus.

\section{Die Gestaltung der Unternehmenskultur - ein weitgehend unbeachtetes Feld}

Die Bedeutung der Unternehmenskultur wird in der Wirtschaftswelt bedauerlicherweise weit unterschätzt. Sie ins Bewusstsein und in den Fokus zu nehmen, kann zum entscheidenden Wettbewerbsvorteil werden. Die Unternehmenskultur dient den grundlegenden Prinzipien und Werten eines Unternehmens und seiner Unternehmensphilosophie. Sie ist so etwas wie der gemeinsame Geist oder auch die Seele eines Unternehmens. Sie zeigt sich vor allem im Kontakt, im Umgang miteinander und der Ausstrahlung der Führungskräfte und Mitarbeiter einer Organisation. Sie macht letztendlich auch ein Unternehmen einzigartig. Und wenn sie authentisch gelebt wird, ist sie auch für seine Kunden, Kooperationspartner und die umgebende Gesell- 
schaft authentisch und damit auch attraktiv. Da es bisher wenig umfassende praktisch anwendbare Modelle zur Entwicklung der Unternehmenskultur gibt, haben wir in den Heiligenfeld Kliniken einmal 7 Prinzipien formuliert und mit erfahrbaren Qualitäten verbunden:

1. Kooperation und Teamgeist, die auf der Qualität der Verbundenheit basieren

2. Gesundheit, sowohl körperlicher als auch psychosozialer Art

3. Inspiration, die über Motivation hinausgeht und auf einer beseelten Haltung zur Arbeit basiert

4. Innovation, die Kreativität voraussetzt

5. Sinn und Spiritualität, die wir durch die Betonung von Achtsamkeit angehen

6. Entwicklung und Lernen, die auf individuelles Wachstum und organisationales Lernen ausgerichtet sind und

7. Führungskunst, die über Management weit hinausgeht und auf einer Verantwortlichkeit basiert.

Für jedes dieser Prinzipien haben wir größere Maßnahmen, die den klassischen Managementinstrumenten entsprechen formuliert und darüber hinaus kleine Gesten implementiert, die kaum etwas kosten, aber die Unternehmenskultur erst richtig lebendig sein lassen. Wenn diese Prinzipien von Führungskräften entsprechend vorgelebt und angeleitet werden, kann eine leidenschaftliche, lebens- und liebenswerte Unternehmenskultur daraus wachsen.

Ein gelebtes Beispiel und gleichzeitig eines unserer stäksten kulturbildenden Elemente ist unsere Veranstaltung zur Organisations-Entwicklung. Hier kommen $1 \mathrm{x}$ in der Woche für 75 Minuten alle therapeutischen Mitarbeiter, alle Mitarbeiter der Verwaltung, aus dem Marketing, der EDV, alle leitenden Mitarbeiter und ausgewählte Mitarbeiter aus den Bereichen Küche, Hauswirtschaft und Facility-Management zusammen. In dieser Großveranstaltung beschäftigen wir uns einerseits mit klassischen Themen, wie z.B. die Vorbereitung auf eine Zertifizierung, Überblick und Ideensammlung für das Marketing, Fehlermanagement oder Kundenorientierung. Andererseits haben wir uns auch intensiv mit dem beschäftigt, was das Wesentliche, die Essenz von Heiligenfeld ausmacht. Wir haben statt eines Leitbildes unsere essenziellen Werte formuliert. Wir haben aber auch nach Trends und Veränderungen gefragt und wie wir darauf reagieren können. Wir haben die Heiligenfelder politischen Positionen einmal formuliert und diskutiert. In dieser Großveranstaltung kommen wir also mit etwa 150-200 Mitarbeitern zusammen und nach einer Erläuterung des Rahmenthemas teilen wir uns in der Regel nach Abteilungen oder abteilungsübergreifenden gemischten Gruppen auf, in denen wir die vorgegebenen Themen diskutieren und Ideen dazu entwickeln. Das Ganze wird protokolliert und systematisch ausgewertet. Einige Vorschläge oder Positionen werden auch beispielhaft in der Großgruppe kommuniziert. Aus solchen Ideen heraus und aus weiteren Impulsen entstehen für einen Teil des Jahres Projektgruppen, die sich für 2-3 Monate innerhalb der gleichen Zeit (dann findet keine Großgruppe statt) in der Regel abteilungsübergreifend zusammengesetzt treffen und spezielle Konzepte und konkrete Umsetzungsvorschläge erarbeiten. Diese Veranstaltung ist ein zentrales Element unserer Unternehmenskulturentwicklung. Sie schafft ein gemeinsames Bewusstsein für das, was uns am Herzen liegt. Sie lässt einen wesentlichen Teil der Mitarbeiter zu Wort und ins Gespräch kommen und nutzt das Potenzial sowohl der leitenden als auch der nicht-leitenden Mitarbeiter zum Erkennen von Veränderungen, Chancen, Gefahren und Schwachstellen des Unternehmens und sie bringt eine Fülle von Ideen und konkreten Vorschlägen hervor, sodass die weitere Entwicklung unseres Unternehmens lebendig bleibt. 
Ich habe dies etwas ausführlicher dargestellt, um zu veranschaulichen, wie es aussehen könnte, die psychosoziale Kompetenz aller Mitarbeiter eines Unternehmens in der Gestaltung der Unternehmenskultur zu entwickeln und zu nutzen.

Kehren wir nach diesem Blick auf unsere organisatorischen und managementbezogenen Kompetenzen für die äußere Gestaltung wieder zurück zur inneren Haltung und der Bedeutung einer Führung, die wirklich im Herzen verankert ist.

\section{Leidenschaftliche Führung}

Welche Qualitäten würde ein „leidenschaftliches Führungsverständnis“ ergänzen?

Für mich bedeutet es eine Bereitschaft, mich wirklich berühren zu lassen, offen zu sein, in gewisser Weise sogar „intim und verletzlich“ mit mir selbst, mit meinem Inneren, was durchaus kräftige und eindeutige Antworten möglich macht. Es heißt auch, immer wieder inne zu halten, Nichtwissen auszuhalten, zu forschen, zu staunen - bereit zu sein für etwas ganz Neues oder Abenteuerliches.

Neben der tief empfundenen Wertschätzung für meine Arbeit und die Menschen mit denen ich co-kreativ zusammenarbeite und die ich führen darf, scheint hier auch eine Qualität auf, die wir selten in unseren Unternehmen benennen: die Liebe.

Sich selbst und den Nächsten lieben zu können, bei gleichzeitiger Anerkennung perspektivischer und sachbedingter Unterschiede, wäre eine gesündere Auseinandersetzungs- bzw. Zusammensetzungs-Basis als die gelebte Realität in unseren Hierarchie- und Kommunikationsstrukturen in unseren Unternehmen.

Leidenschaftliches Führen beinhaltet also eine Fähigkeit, mit der eigenen Tiefe in Kontakt zu kommen und zu bleiben, die Kompetenz an Präsenz und Co-Präsenz zu stärken. Leichter ist es natürlich in Glanzmomenten: wenn die Bilanzen stimmen, die Ziele erreicht sind, der Mitarbeiter, mit dem ich gerade spreche, mir sowieso sympathisch ist. Und genau dann können wir beginnen, die Seele zu öffnen, diese Geschenke wirklich tief bei uns ankommen zu lassen. Die natürlichen Antworten unserer Seele sind Freude und Dankbarkeit für diese Erfolge, für diese Beziehungen und unsere Verbundenheit.

So beginnen wir mit dem Üben immer - im leichtesten Level.

Level 2 könnte heißen: Ich höre meine Seele auch dann, wenn es langweilig wird, in meinen Routinen oder am Montagmorgen. Die Kette der Steigerungen führt uns über den Umgang mit Ambivalenzen und Unsicherheiten bis in emotionale Konfliktgespräche. Was sagt meine Seele zu diesem „ewigen Fehlverhalten“ meiner Mitarbeiter? Und wie kann ich im Gespräch meine eigenen Perspektiven und Fühlrichtungen frei wählen statt ihnen ausgeliefert zu sein? Um hier von einen Kennen in der Theorie in ein elegantes Können einzutauchen, braucht es viel Übung und viel Praxis. Jede unbequeme Situation bietet wieder eine neue Gelegenheit dafür. Für mich sind nicht mehr nur die Momente des tiefen Erkennens gemeinsamer Arbeit in den Augen der anderen meine Highlights, sondern auch ein würdevoll geführtes Trennungsgespräch, indem wir statt über hohe Abfindungssummen zu streiten, unsere unterschiedlichen Werte und Perspektiven auseinandermischen und ein für beide Seiten „stimmiges Ende“ finden.

Leidenschaftliche Augenblicke erlebe ich auch, wenn eine Azubi mir im Gespräch auf behutsames Nachfragen hin anvertraut, dass ihre Verletzungen beim Essenzu- 
bereiten in unserer Küche nicht aus Ungeschicklichkeit stammen, sondern weil sie permanent innerlich im Computerspiel „world of warcraft“ lebt, um der äußeren Realität und ihrer übergriffigen Mutter zu entfliehen.

Oder wenn wir eine ernsthafte Diskussion um die erlaubte Hosenbeinlänge der Physiotherapeuten im Sommer führen, weil die psychosomatischen, evtl. sexuell traumatisierten Patientinnen sich davon belästigt fühlen könnten. Hier geht es nicht nur um die Ergebnisse der Gespräche, sondern v.a. um einen Bewusstwerdungsprozess. Wenn verborgene Haltungen und persönliche Wahrheiten bewusst werden dürfen und noch mehr, wenn sie ausgedrückt und in den Kontakt kommen dürfen, dann finden sich viel leichter angemessene Lösungen.

In der Führungspraxis von Teams gilt es immer wieder, die Fähigkeit des Perspektivwechsels anzuleiten und zu nutzen, um schnellen oder einseitigen Lösungen vorzubeugen. Diese Flexibilität und innere Beweglichkeit ermöglicht mehr Weite und fühlt sich von den Beteiligten oft als sehr lebendig und ausgeglichen an. Je mehr TeamTeilnehmer über diese Qualität verfügen, desto entspannt-leidenschaftlicher wird der Prozess des Aushandelns, denn es geht nicht mehr um Gewinnen oder Verlieren, sondern um die für das Ganze beste Lösung zu diesem Zeitpunkt.

\section{Die Herausforderung des Geführt-werdens}

Und was gehört noch zum leidenschaftlichen Führen? Ganz bestimmt das „Geführt werden“. Wie oft habe ich schon gehört: „Wer sich nicht durch andere führen lassen kann, kann auch nicht andere führen!“ Dieser Imperativ spielt u.a. für die Balancierung der Seele eine größere Rolle als wir zunächst glauben. In der Auseinandersetzung mit unserer Geschichte der „Geführt-werdens“ müssen wir uns eingestehen, dass wir unsere eigenen Führungserlebnisse durch Eltern, Lehrer, Chefs oft unhinterfragt weitertragen und wiederholen oder in Abgrenzung dazu ganz anders machen wollen - was ebenfalls nicht frei ist. Für eine liebevolle Selbstführung brauchen wir in uns selbst beide Aspekte: einen führenden und einen geführten Anteil. Manchmal erlebe ich meine Seele als die größere, umfassendere Instanz, die meine Persönlichkeit an der Hand nimmt und sie leitet, statt dass ich mich von den Verführungen und Sehnsüchten meiner Persönlichkeit bestimmen lasse.

Dort, wo mir das nicht gelingt, an Plätzen der Reibung, des Narzissmus, der Verlockungen der Oberfläche, bin ich dankbar für einen ,äußeren Lehrer“ oder Begleiter, der als Anwalt meiner Seele agiert. Ihm kann und darf ich mich dann anvertrauen in meinen Zweifeln, in meiner Suche, ohne dass er mich berät oder therapiert. Er bezeugt mein Leben, hält es in seinem offenen Gewahrsein und unterstützt meine Seelenbewegungen. Für unser Handwerk brauchen wir Führungskräfte manchmal auch einen Berater oder eine Beraterin in Fragen des Managements, der Mitarbeiterführung, der Strategie. Aber wirklicher Luxus sind für mich die Begleiter, die sich mit mir ins Unbekannte wagen, die dort forschen und spiegeln, wo ich etwas nicht weiß, wo ich beginne mich zu überschreiten, mich zu riskieren. Wo ich auch scheitern darf.

Bei allem Wissen um die Weiterentwicklung zur eigenen inneren Selbstführung tut mir diese auch physisch empfundene Rolle der Schülerin einfach gut. Ich empfinde sie weitgehend nicht als kindlich oder übertragend, sondern als echte Balancierung, als Rückhalt, Stärkung und Seelenluxus. Auch als langjährige, tiefe oder spirituelle 
Freunde können wir uns diesen Liebesdienst erweisen, Konfrontationen eingeschlossen. Wer von uns das Glück eines Austauschpartners oder einer Freundin mit Führungsverantwortung hat, sollte dieses Potenzial unbedingt würdigen und nutzen. Nicht nur zur gegenseitigen Bestätigung und Entlastung, sondern v.a. zur gegenseitigen Befragung. Was sind Deine tiefsten Werte in der Führung? Was gibt Dir jeden Morgen die Kraft aufzustehen und Deine Aufgabe zu erfüllen? Wie gehst Du mit persönlich mit Deinem Geld um? Wie geht es Dir körperlich, seelisch und in Deinen Beziehungen? Wo gibt es bei Dir Abweichungen zwischen „ich will/sollte“ und dem „ich tue das"? Und wie willst Du diese Lücke schließen? Die Manager unter uns könnten ja aus diesem Check ein Protokoll, eine „To-do“-Liste und eine Wiedervorlage erstellen, was ich übrigens für sehr nützlich halte. Wenn ich mir und meinem Gegenüber beim fünften Check immer noch die $10 \mathrm{~kg}$ zu viel, die schlaflosen Nächte oder das wachsende Minus auf meinem Privatkonto eingestehe, ist es Zeit für eine tiefere Sondierung über meine „Kunst mich selbst zu boykottieren“. Vielleicht finden wir gemeinsam ein tieferes Erkennen und Verstehen und neue kreative Wege, die Leidenschaft zur Umsetzung persönlicher Ziele zu wecken. Eine Führung in die Transzendenz kann nie von außen geschehen. Manchmal wirkt eine Freundschaft, ein Lehrer- oder Meister-/Schülerverhältnis so, aber die tiefste Tür zu unserer Quelle, zum Ursprung, zur Unmittelbarkeit des Lebens öffnen wir selbst.

Ein Außen kann helfen, gute Bedingungen herzustellen, kann inspirieren und einladen, die Öffnung aber nicht bewirken. Diese tiefste Öffnung ist ein Moment leidenschaftlicher Hingabe und Selbstüberschreitung. Hier entlässt sich die Seele aus der Selbstführung und übergibt sich an eine größere Weisheit oder Intelligenz. So könnten wir die eben ausführlich beschriebene Seelen-Führung als Zwischenstadium zur kosmischen oder umfassenden Führung sehen, aber von dieser schätze ich uns realistischerweise noch weiter entfernt ein.

\section{Wie weit kann bewusste Führung reichen?}

Wenn unsere globale Menschheits-Seele den Ruf der universellen Intelligenz hören und leben könnte, wären die Gestaltungen und Unternehmungen auf unserer Erde wahrscheinlich andere. Ich meine das durchaus nicht idealistisch, sondern als Ahnung eines Potenzials, vielleicht als idealistischen Realismus im Sinne Rilkes Aussage:

„Ich lebe mein Leben in wachsenden Ringen, die sich über die Dinge ziehen. Ich werde den letzten vielleicht nicht vollbringen, aber versuchen will ich ihn."

Wenn wir uns in die Mitte unserer Führungsaufgabe stellen, mit unserem Potenzial, unseren Schwächen, unserer Reichweite und unseren Grenzen; wenn wir das alles wirklich zu uns nehmen, welche Bewegung entsteht daraus?

Vielleicht gelingt es uns dann, mit unserem gesamten Wissen über Management, Leadership und Strategien einzutauchen in etwas uns jetzt noch ganz Unbekanntes; das Wagnis zu gehen, Verbundenheit, Bewusstsein und Liebe im Arbeitsalltag zu benennen und v.a. zu leben. Vielleicht werden wir demütig angesichts der Größe und Schönheit des Lebens und beginnen unsere Führungsaufgabe mehr und mehr als Dienerschaft zu verstehen. Oder noch poetischer: wir empfinden uns wie eine Geliebte oder ein Geliebter im Tanz mit der kosmischen Intelligenz, der wir unser eigenes Potenzial und unsere Schönheit zur Verfügung stellen. 
Für uns als sich auf dem Weg befindenden Führungskräfte könnten die Leidenschaften und Sehnsüchte unserer Seele eine gute Spur legen, der Erfüllung näher zu kommen - wie Hans-Peter Dürr auf dem Kongress der Akademie Heiligenfeld zum Thema „Bewusstsein“ es so wundervoll formulierte: „Freiheit heißt dann nicht: Lieber Gott schraub mir meine Ethik ab, damit ich mich freier bewegen kann, sondern der Wahrhaftigkeit ganz, ganz nah kommen“, nicht indem wir den Objekten unserer Leidenschaften folgen, sondern indem wir selbst leidenschaftlich werden und unsere Führungsverantwortung leidenschaftlich leben und lieben.

\section{Literatur}

Brown B (2004) Befreiung vom inneren Richter, Kamphausen Verlag 2004, ISBN 3-933496-04-7

Dürr H-P (2013) Wir erleben mehr als wir begreifen. Plenarvortrag auf dem Kongress „Bewusstsein“ der Akademie Heiligenfeld in Bad Kissingen Juni 2013, Auditorium Netzwerk, Audio und DVD

Menhuin Y (1987) Musik kann man nur hören, Beitrag aus „zeitmagazin“ Hamburg 1987

Rilke RM 20.09.1899 Berlin-Schmargendorf

Schulz von Thun F (2000) Der innenpolitische Sprecher der SPD-Bürgerschaftsfraktion, Ingo Kleist, zum idealen Profil eines neuen Polizeipräsidenten für Hamburg.

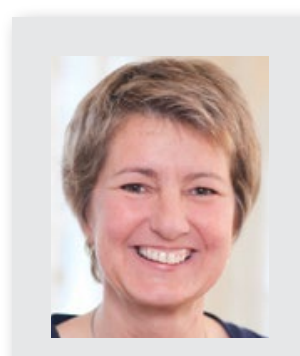

\section{Dorothea Galuska}

Leiterin des Personalmanagements der Heiligenfeld Kliniken, langjährige therapeutische Leiterin der Heiligenfeld Kliniken, Physio-, Bewegungs- und Körperpsychotherapeutin mit Ausbildungen in integrativer Bewegungstherapie und Orgodynamik, Europäisches Zertifikat für Psychotherapie (ECP), Meditationslehrerin, Schülerin der Ridhwan School (Almaas) 